ITEP-LAT/2001-1

KANAZAWA-01-11

15 October, 2001

\title{
Monopoles and hybrids in Abelian projection of lattice QCD *
}

\author{
P.Yu. Boyko ${ }^{a}$, M.N. Chernodub ${ }^{\mathrm{a}, \mathrm{b}}$, A.V. Kovalenko ${ }^{\mathrm{a}}$, S.M. Morozov ${ }^{\mathrm{a}}$, M.I. Polikarpov ${ }^{\mathrm{a}}$ \\ ${ }^{a}$ Institute of Theoretical and Experimental Physics, B.Cheremushkinskaya 25, Moscow, 117259, Russia \\ bInstitute for Theoretical Physics, Kanazawa University, Kanazawa 920-1192, Japan
}

We study topological defects constructed from diagonal and non-diagonal gluons in Abelian projection of zero temperature lattice QCD. We compare results obtained in the quenched and non-quenched vacuum field configurations and show that the density of hybrids is higher than the density of monopoles. The density of some hybrids is sensitive to the presence of the virtual fermions.

\section{INTRODUCTION}

There are a lot of numerical facts indicating that Abelian monopoles in the Maximal Abelian (MA) projection of lattice gluodynamics play an important role in the confinement scenario (see, e.g., reviews [1]). The monopoles are constructed from diagonal elements of the gluon field matrix, these elements corresponds to the gauge field in the Abelian projection [2]. The off-diagonal gluons play the role of the electrically charged matter fields.

Besides the Abelian monopoles one can construct 3] other topologically stable defects ("hybrids") using both diagonal and off-diagonal gluons in $S U(2)$ lattice gauge theory.

In the present publication we generalize the results of Ref. [3] to the $S U(3)$ gauge group and calculate the density of hybrids in quenched and non-quenched lattice QCD.

\section{DEFINITION OF HYBRIDS}

The monopole current for $S U(2)$ lattice gauge theory is defined as follows:

${ }^{*} j=\frac{1}{2 \pi} * \mathrm{~d}(\mathrm{~d} \theta \bmod 2 \pi)$,

here $\theta$ is the phase of the diagonal element of the link matrix $U_{i i}=\left|U_{i i}\right| \exp i \theta_{i}$, and we use the notations of lattice differential forms: $\mathrm{d} \theta$ is the standard plaquette angle, "d" is a differential operator on the lattice and the current ${ }^{*} j$ is

\footnotetext{
${ }^{*}$ Presented by M.I.P. at Lattice 2001, Berlin.
}

an integer-valued one form (4-vector) on the dual lattice.

The current defined by eq. (11) is

a) invariant under the $U(1)$ gauge transformations: $\theta \rightarrow \theta+d \alpha, j \rightarrow j$.

b) topologically stable: a small (but finite) variation of $\theta$ does not change the current $j$.

c) integer-valued, ${ }^{*} j_{x, \mu} \in \mathbb{Z}$.

d) conserved, $\delta^{*} j=\delta^{*} \mathrm{~d}(\mathrm{~d} \theta \bmod 2 \pi)=0$, due to the identities $\mathrm{d}^{2} \equiv 0$ and $\mathrm{d} \equiv{ }^{*} \delta^{*}$.

The generalization of the above construction to $S U(3)$ gauge group was done in Ref. 顿. Below we construct the hybrid $S U(3)$ currents, satisfying conditions $a)-d$ ).

Condition $a$ ) demands the gauge invariance of the hybrid currents under the $U(1) \times U(1)$ Abelian gauge transformations, $U_{x, \hat{\mu}} \rightarrow T_{x} U_{x, \hat{\mu}} T_{x+\hat{\mu}}^{\dagger}$, where the matrix of the Abelian gauge transformation is defined as follows:

$T_{x}=\left(\begin{array}{ccc}e^{i \alpha_{x}} & 0 & 0 \\ 0 & e^{i \beta_{x}} & 0 \\ 0 & 0 & e^{-i\left(\alpha_{x}+\beta_{x}\right)}\end{array}\right)$.

It is easy to see that under this transformation the phases of the link matrix are transformed as shown in Table 1.

For simplicity we use the "naive" definition of the monopole current for $S U(3)$ gauge group,

${ }^{*} j_{i}=\frac{1}{2 \pi} \mathrm{d}\left(\mathrm{d} \theta_{i} \bmod 2 \pi\right)$. 
Table 1

The $U(1) \times U(1)$ gauge transformation.

\begin{tabular}{ll}
\hline Field & Transformed field \\
\hline$\theta_{1}$ & $\theta_{1}+\alpha_{x}-\alpha_{x+\hat{\mu}}$ \\
$\theta_{2}$ & $\theta_{2}+\beta_{x}-\beta_{x+\hat{\mu}}$ \\
$\theta_{3}$ & $\theta_{3}-\left(\alpha_{x}-\alpha_{x+\hat{\mu}}\right)-\left(\beta_{x}-\beta_{x+\hat{\mu}}\right)$ \\
$\chi_{12}$ & $\chi_{12}+\alpha_{x}-\beta_{x+\hat{\mu}}$ \\
$\chi_{21}$ & $\chi_{21}+\beta_{x}-\alpha_{x+\hat{\mu}}$ \\
$\chi_{13}$ & $\chi_{13}+\alpha_{x}+\alpha_{x+\hat{\mu}}+\beta_{x+\hat{\mu}}$ \\
$\chi_{31}$ & $\chi_{31}-\alpha_{x}-\alpha_{x+\hat{\mu}}-\beta_{x}$ \\
$\chi_{23}$ & $\chi_{23}+\beta_{x}+\beta_{x+\hat{\mu}}+\alpha_{x+\hat{\mu}}$ \\
$\chi_{32}$ & $\chi_{32}-\beta_{x}-\beta_{x+\hat{\mu}}-\alpha_{x}$ \\
\hline
\end{tabular}

It occurs that the results for the monopole current density obtained with the "naive" definition (2) coincide with $10 \%$ accuracy with the results obtained using the "accurate" definition given in Ref. 沺 (see also the contribution of T. Streuer in these Proceedings). The definition of the hybrid current is analogous to that of the monopole current. First we construct the $U(1) \times U(1)$ gauge invariant plaquette variables $p_{m}$ from $\theta_{i}$ and $\chi_{i j}$, these are the analogues of $d \theta_{i}$. Then we define the plaquette angle $P_{m}$ :

$P_{m}=\sum_{k}\left(p_{k} \bmod 2 \pi\right)$,

As an example, we show the construction of $P_{m}(H 1)$ variable explicitly:

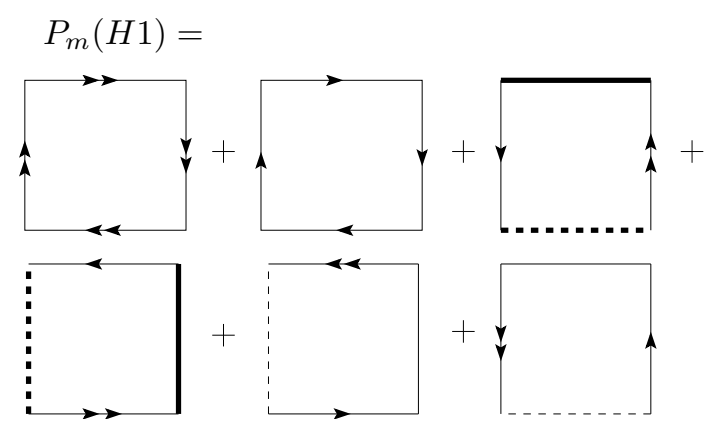

Here the variables $\theta$ are shown by solid lines with arrows; the variables $\chi$ are shown by arrows; the variables $\chi$ are shown by the solid lines and the variables $-\chi$ are shown by the dashed lines. Different species of $\theta$ 's differs from each other by the number of arrows while the species of $\chi$ are distinguished by the width of the lines.

The 3 -forms satisfying condition $c$ ) can be constructed from the 2 -forms $P_{m}\left(\theta_{i}, \chi_{i, j}\right)$ attached to six faces of the $3 D$ cube,

${ }^{*} j_{H 1}=\sum_{m=1}^{6} P_{m}(H 1)$.

We construct $J_{H 1}$ in such a way that the hybrids disappear in non-compact theory (if we disregard $\bmod 2 \pi$ in eq. (3)). Thus the hybrids are topologically stable objects (i.e., condition $b$ ) is satisfied). In a separate publication we will show that $\delta j_{H 1}=0$. Thus condition $d$ ) is satisfied for $j_{H 1}$ as well.

There are a lot of types of currents $j_{H}$ which can be constructed from $\theta_{i}$ and $\chi_{i j}$. However, in the MA projection one can observe a natural hierarchy of the hybrid currents. Indeed, the MA projection implies the maximization of the modulus of the diagonal elements, $U_{i i}$, of the link matrix $U$. Therefore the modulus of the off-diagonal elements $U_{i j}$ is minimized and, as a result, the diagonal elements are most important for the dynamics. Since the ordinary monopoles are extracted from diagonal elements, $U_{i i}$, they are the most important topological defects.

To construct monopoles we use the plaquette angles,

$$
P_{m}\left(\theta_{i}, \chi_{i j}\right)=p_{m}\left(\theta_{i}\right) \bmod 2 \pi=p_{m}^{(0)} .
$$

There are $36 U(1) \times U(1)$ invariant plaquettes $p_{m}^{(2)}$ constructed from two variables $\theta_{i}$ and two variables $\chi_{i j}$ : there exist $24 U(1) \times U(1)$ invariant plaquettes $p_{m}^{(3)}$ constructed from one $\theta_{i}$ and three variables $\chi_{i j}$ etc. We expect that hybrids constructed from $p_{m}^{(2)}$ are more important for dynamics than those constructed from $p_{m}^{(k)}, k=3,4$. It occurs that there are 3 conserved hybrids $H 1, H 2, H 3$ constructed from $p_{m}^{(0)}$ and $p_{m}^{(2)}$. The construction of $H 1$ is outlined above and the construction of $H 2$ and $H 3$ hybrids is similar. 


\section{NUMERICAL RESULTS}

We have calculated the density of hybrids $H 1, H 2, H 3$ using vacuum fields [6] generated in quenched and non-quenched lattice $S U(3)$ QCD on the lattice $16^{3} \times 32$. The non-quenched configurations are generated for $O(a)$ improved Wilson fermions, the parameters of simulation are: $\beta=5.26, \kappa_{\text {sea }}=0.1345$, the other parameters are given in Ref. [5]. The quenched configurations are generated for $\beta=6.0$. This value of $\beta$ is chosen in such a way that the force between heavy quarkantiquark pair at the distance $r_{0}=0.52 \mathrm{fm}$ is the same in quenched and non-quenched case.

Our numerical results are given in Table 2. The first raw represents the density of monopoles (see talk of T.Streuer on this symposium), the other lines correspond to the density of hybrids $H 1, H 2, H 3$ discussed in the previous section. The last column represents the density of the corresponding hybrids generated in random ensembles of Abelian fields.

One can draw a few conclusions from Table 2. The monopole density is much lower than the corresponding random value, while the hybrids are much more randomized. The density of $H 1$ hybrid is sensitive to the presence of the virtual quarks. Note, however, that the difference in the densities of hybrids in quenched and nonquenched vacuum is much smaller than the similar difference for monopoles.

Table 2

The density of the monopoles and hybrids in the quenched and non-quenched QCD and in random ensembles.

\begin{tabular}{llll}
\hline & quenched & non-quenched & random \\
\hline M & $0.0062( \pm 4)$ & $0.0153( \pm 5)$ & $0.5023( \pm 4)$ \\
\hline H1 & $0.1049( \pm 3)$ & $0.1136( \pm 3)$ & $0.2241( \pm 1)$ \\
H2 & $0.1219( \pm 1)$ & $0.1245( \pm 2)$ & $0.2283( \pm 3)$ \\
H3 & $0.1545( \pm 2)$ & $0.1551( \pm 3)$ & $0.3078( \pm 4)$ \\
\hline
\end{tabular}

\section{NOT RESOLVED QUESTION}

We do not know what magnetic and electric charge carry the hybrids $H 1, H 2$ and $H 3$. It is important to find a linear combination of the hybrid currents which corresponds to a pure electric current. This current can be rather sensitive to the presence of the dynamical fermions.

\section{ACKNOWLEDGMENTS}

The authors are grateful to F.V. Gubarev, G. Schierholz, T. Streuer, T. Suzuki and V.I. Zakharov for useful discussions. M.I.P is partially supported by grants RFBR 00-15-96786, RFBR 01-02-17456, INTAS 00-00111 and CRDF award RP1-2103. M. N. Ch. is supported by JSPS Fellowship P01023. P.Yu.B., A.V.K. and S.M.M. are very grateful to Physics Department of Kanazawa University for financial support on summer 2001.

\section{REFERENCES}

1. R. W. Haymaker, Phys. Rept. 315 (1999) 153; G. S. Bali, hep-ph/9809351; M. N. Chernodub and M. I. Polikarpov, hep-th/9710205.

2. G. 't Hooft, Nucl. Phys. B190 (1981) 455.

3. M. N. Chernodub, M. I. Polikarpov and A. I. Veselov, Phys. Lett. B342 (1995) 303; E.T. Akhmedov, M.N. Chernodub and M.I. Polikarpov, RCNP Confinement 1995, p. 37, hep-lat/9504013.

4. A.S. Kronfeld, G. Schierholz and U.J. Wiese Nucl.Phys. B293 (1987) 461; M.I. Polikarpov and Ken Yee, Phys.Lett. B316 (1993) 333.

5. S. Booth et. al, hep-lat/0103023.

6. We are grateful to T. Streuer and G. Schierholz, who provide us with $S U(3)$ gauge field configurations. 